\title{
Prevalence and characteristics of ST131 clone among unselected clinical Escherichia coli in a Chinese university hospital
}

\author{
Bin Li', Yanfang Lư ${ }^{2}$, Fangjun Lan'1 Q Qingwen $\mathrm{He}^{1}$, Chen $\mathrm{Li}^{3}$ and Yingping $\mathrm{Cao}^{1 *}$
}

\begin{abstract}
Background: Escherichia coli clinical sequence type 131 (ST131) has emerged as an extensively antimicrobial resistant E. coli clonal group in recent years throughout the world. The aim of this study was to investigate the prevalence and molecular characteristics of ST131 among unselected E. coli clinical isolates in a Chinese university hospital.

Methods: Seven hundred consecutive E. coli isolates were collected at a Chinese university hospital between 2014 and 2015. Isolates belonging to ST131 were identified by PCR and multilocus sequence typing (MLST), and then characterized for antibiotic resistance, CTX-M-type extended-spectrum $\beta$-lactamase genes, fluoroquinolone resistance genes, $O$ types, phylogenetic groups, virulence factors and PFGE patterns.

Results: Overall, 83 (11.6\%) isolates were identified as ST131 group. The H30 lineage accounted for 53 (63.9\%) of the ST131 isolates, including 13 H30-Rx and 40 H30 non-Rx. The remaining 30 isolates belonged to H41 lineage. Two O types were identified in this study: O25b (66.3\%) and O16 (33.7\%). Compared with O25b-B2-ST131 isolates, O16-B2-ST131 isolates harbored less virulence factors of adhesins. ST131 H30 Rx isolates had significantly higher virulence score than those of other isolates. O16-B2-ST131 isolates were shown to have a lower resistance to quinolones than O25b-B2-ST131 isolates. 5 nonsynonymous mutations (GyrA S83 L, D87N, ParC S80I, E84V and ParE I529L) were strongly associated with ST131 H30 and O25b isolates. Results of PFGE demonstrated that these isolates were classified into 68 pulsotypes and these subtypes were grouped into 23 different PFGE clusters using 70\% similarity cut-off value.

Conclusions: This is the first study to reveal the prevalence and molecular characteristic of ST131 clonal group among consecutive clinical E. coli isolates in China. Our findings demonstrated that ST131 lineage accounts for a small proportion of clinical E. coli isolates in China, which included two major groups: O25b-B2-ST131 and O16-B2-ST131. Our results implies that O16-B2-ST131 subclone may be an important type of E. coli ST 131 in China, which suggests that future studies should not ignore such clone in this country.
\end{abstract}

Keywords: E. coli, ST131, Phylogenetic group, O256, O16, H30

\section{Background}

Escherichia coli sequence type 131 (E. coli ST131) was identified among extended-spectrum $\beta$-lactamases (ESBL)producing isolates in Asia, Europe and North America in 2008, and has rapidly emerged globally to become an important pathogen causing various infections in humans $[1,2]$. E. coli ST131 is becoming the predominant

\footnotetext{
*Correspondence: caoyingping918@hotmail.com

'Department of Clinical Laboratory, Fujian Medical University Union Hospital,

29 Xinquan Rd, Fuzhou, Fujian 350001, People's Republic of China

Full list of author information is available at the end of the article
}

extraintestinal pathogenic E. coli clone which drives multidrug resistance [1].

Although some of E. coli ST131 isolates are nontypeable for $\mathrm{O}$ antigen, most of these strains are of serotypes $\mathrm{O} 25 \mathrm{~b}$ and $\mathrm{O} 16$ [1, 2]. All E. coli ST131 isolates contain the fimH gene with high level of allelic diversity and fimH30 is the most common one [1]. H30 lineage of ST131 is named after fimH30 allele and includes two important sublineages (H30-R and H30-Rx) [1-3]. E. coli ST131 isolates are often resistant to fluoroquinolones and produce ESBL [4-6]. The $H 30$ subclone comprises most of the fluoroquinolone-resistant ST131 isolates [4]. 
The main mechanism of fluoroquinolones resistance in E. coli ST131 isolates is aminoacid substitutions in the quinolone-resistance determining region (QRDR) of gyrA, parC and parE $[1,2,6]$. Within the $H 30$ subclone, the $H 30-\mathrm{Rx}$ subset is strongly associated with CTX-M $\beta$ lactamases $[1,2]$.

Regarding the spread of $E$. coli ST131, most investigations have focused on antimicrobial-resistant isolates, especially those resistant to fluoroquinolones and cephalosporins $[1,2]$. E. coli ST131 has been detected among extendedspectrum $\beta$-lactamase-producing or fluoroquinoloneresistant isolates in China [7-9]. However, the prevalence of ST131 among unselected E. coli isolates remains unknown. The aim of this study was to investigate the prevalence and molecular characteristics of ST131 in a collection of 700 unselected clinical E. coli isolates collected at a university hospital in Southern China.

\section{Methods}

Bacterial isolates and screening for ST131

A total of 700 non-duplicate $E$. coli clinical isolates were consecutively collected at a Chinese university hospital (Fujian Medical University Union Hospital, Fuzhou, Fujian province, China) between August 2014 and August 2015. These isolates were obtained from urine (51.0\%), blood (14.7\%) and miscellaneous sources (34.3\%). Isolates were identified by the Vitek-2 system GNI card (BioMèrieux, Missouri, France). All isolates were screened for ST131 by PCR-based method for ST131-associated SNPs in $m d h$ and $\operatorname{gyr} B$ as previous described [10]. All the non-O25b, non-O16 and non-phylogenetic group B2 ST131 strains would be confirmed by multilocus sequence typing (MLST) to be ST131 according to the Achtman scheme using seven housekeeping genes ( $a d k$, fum $C, \operatorname{gyr} B$, icd, $m d h$, purA and recA) (http://mlst.ucc.ie/mlst/dbs/Ecoli). All ST131 isolates detected were further tested by PCR using specific primers for the fim $\mathrm{H} 30$ allele for identification of the $H 30$ subclone [11]. The $H 30-\mathrm{Rx}$ subclone was identified using allele-specific PCR as previously described [11]. All the H30-PCR negative isolates underwent direct sequenceing of $f i m H$ as previously described [3].

\section{Susceptibility testing}

Antimicrobial susceptibility of E. coli ST131 isolates was determined by disk diffusion [12]. Drugs tested included aztreonam (ATM), cefotaxime (CTX), ceftazidime (CAZ), cefepime (FEP), ertapenem (ETP), imipenem (IPM), piperacillin-tazobactam (TZP), ciprofloxacin (CIP), levofloxacin (LEV), amikacin (AMK), and trimethoprimsulfamethoxazole (SXT). The results were interpreted according to the breakpoints of the 2016 CLSI criteria [12]. E. coli ATCC 25922 was used for routine quality control.

\section{Molecular characterization of ST131}

Phylogenetic groups were determined using PCR method described by Clermont et al. previously [13]. Molecular O types were performed on all ST131 isolates by PCR with primers as described previously [14]. The ST131-associated O25b rfb variant was detected by a separated PCR assay [15]. The presence of 26 virulence factors (VFs) genes was evaluated by a multiplex PCR method [16].

The virulence score (VF score) was calculated for each isolate as the sum of all virulence-associated genes detected in this study [17]. The sum of all the VF scores of the isolates was then calculated, and finally this sum was divided by the number of isolates to give the mean VF score. Pap and $s f a-f o c$ were counted only once regardless of the number of elements or subunits identified [17].

\section{Detection of bla $a_{\mathrm{CTX}-\mathrm{M}}$ gene and fluoroquinolone resistance genes}

Isolates nonsusceptible to cefotaxime or ceftazidime underwent detection of bla $a_{\text {CTX-M }}$ using PCR [18]. As to CIPresistant isolates, mutations in quinolone-resistance determining region (QRDR) of gyrA, parC and parE were determined by PCR and sequencing [19]. Regarding CIP-nonsusceptible or bla $a_{\mathrm{CTX}-\mathrm{M}}$ producing isolates, the presence of plasmid mediated quinolone resistance determinants (PMQRs; qnrA, qnrB, qnrC, qnrD, qnrS, and $\left.a a c\left(6^{\prime}\right)-I b-c r\right)$ were detected by PCR as previously described [20, 21].

\section{Pulsed-field gel electrophoresis analysis (PFGE) and dendrogram construction}

ST131 isolates were subjected to PFGE analysis using XbaI digestion [18]. A PFGE dendrogram was constructed with BioNumerics software (Applied Maths, Sint-MartensLatem, Belgium) according to the unweighted pair group method based on Dice coefficients. Isolates with a Dice similarity index $\geq 70 \%$ were considered to belong to the same PFGE cluster.

\section{Statistical analysis}

Data were analyzed using the SPSS 19.0. Comparisons of proportions were performed using $X^{2}$ or Fisher's exact test (two tailed) or Mann-Whitney test. For each comparison, $p<0.05$ was considered to be statistically significant.

\section{Results}

\section{Prevalence of ST131 and its subclones}

In total, $83(11.6 \%)$ of the 700 clinical $E$. coli isolates were identified as ST131. ST131 accounted for $15.4 \%$ $(55 / 357)$ of the urinary E. coli isolates and $11.7 \%(12 /$ 103) of the bloodstream isolates. E. coli ST131 strains were isolated from patients from 23 different wards. Females accounted for $60.2 \%(50 / 83)$ of the ST1193 
Table 1 Characteristics of E. coli ST131 isolates by fimH subtyping

\begin{tabular}{|c|c|c|c|c|c|c|c|}
\hline \multirow[t]{3}{*}{ Characteristics } & \multirow{3}{*}{$\begin{array}{l}\text { Total } \\
N=83(\%)\end{array}$} & \multicolumn{3}{|c|}{ ST131 subclones, number of isolates (\%) } & \multicolumn{3}{|l|}{$P^{\mathrm{a}}$} \\
\hline & & \multirow{2}{*}{$\begin{array}{l}\text { H3ORx } \\
n=13\end{array}$} & \multirow{2}{*}{$\begin{array}{l}\text { H30 non-Rx } \\
n=40\end{array}$} & \multirow{2}{*}{$\begin{array}{l}\mathrm{H} 41 \\
n=30\end{array}$} & \multirow[t]{2}{*}{$\begin{array}{l}\text { H41 vs } \\
\text { H30 non -Rx }\end{array}$} & \multirow[t]{2}{*}{$\begin{array}{l}\text { H41 vs } \\
\text { H30-Rx }\end{array}$} & \multirow[t]{2}{*}{$\begin{array}{l}\text { H30 Rx vs } \\
\text { H30 non-Rx }\end{array}$} \\
\hline & & & & & & & \\
\hline \multicolumn{8}{|l|}{ PCR O type } \\
\hline O25b & $55(66.3 \%)$ & $12(92.3 \%)$ & $40(100 \%)$ & $3(10.0 \%)$ & $<0.001$ & $<0.001$ & \\
\hline 016 & $28(33.7 \%)$ & $1(7.7 \%)$ & 0 & $27(90.0 \%)$ & & & \\
\hline \multicolumn{8}{|l|}{ Antimicrobial resistance } \\
\hline ATM & $30(36.1 \%)$ & $12(92.3 \%)$ & $11(27.5 \%)$ & $7(23.3 \%)$ & & $<0.001$ & $<0.001$ \\
\hline CAZ & $22(26.5 \%)$ & $10(76.9 \%)$ & $5(12.5 \%)$ & $7(23.3 \%)$ & & 0.002 & $<0.001$ \\
\hline CTX & $59(71.1 \%)$ & $12(92.3 \%)$ & $28(70 \%)$ & $19(63.3 \%)$ & & & \\
\hline FEP & $26(31.3 \%)$ & $11(84.6 \%)$ & $10(25.0 \%)$ & $5(16.7 \%)$ & & $<0.001$ & $<0.001$ \\
\hline TZP & $1(1.2 \%)$ & 0 & 0 & $1(3.3 \%)$ & & & \\
\hline CIP & $58(69.9 \%)$ & 13(100\%) & $40(100 \%)$ & $5(16.7 \%)$ & $<0.001$ & $<0.001$ & \\
\hline LEV & $58(69.9 \%)$ & 13(100\%) & $40(100 \%)$ & $5(16.7 \%)$ & $<0.001$ & $<0.001$ & \\
\hline AMK & $7(8.4 \%)$ & $3(23.1 \%)$ & $1(2.50 \%)$ & $3(10.0 \%)$ & & & 0.042 \\
\hline SXT & $56(67.5 \%)$ & 10(76.9\%) & $24(60.0 \%)$ & $22(73.3 \%)$ & & & \\
\hline \multicolumn{8}{|l|}{ bla $a_{\text {CTX-M }}$ type } \\
\hline CTX-M-14 & $37(44.6 \%)$ & 0 & $22(55.0 \%)$ & 15(50.0\%) & & 0.001 & $<0.001$ \\
\hline CTX-M-15 & $22(26.5 \%)$ & $12(92.3 \%)$ & $7(17.5 \%)$ & $3(10.0 \%)$ & & $<0.001$ & $<0.001$ \\
\hline CTX-M-14,15 & $2(2.40 \%)$ & 0 & $2(5.0 \%)$ & 0 & & & \\
\hline CTX-M-123 & $1(1.2 \%)$ & 0 & 0 & $1(3.3 \%)$ & & & \\
\hline No ESBL ${ }^{b}$ & $21(25.3 \%)$ & $1(7.7 \%)$ & $11(27.5 \%)$ & $9(30.0 \%)$ & & & \\
\hline \multicolumn{8}{|l|}{$\mathrm{FQ}^{\mathrm{R}}$ genes $^{c}$} \\
\hline 5 gyrA, parC and parE mutations ${ }^{d}$ & $51(61.4 \%)$ & $13(100 \%)$ & $35(87.5 \%)$ & $3(10.0 \%)$ & $<0.001$ & $<0.001$ & \\
\hline$a a c\left(6^{\prime}\right)-1 b-c r$ & 14(16.9\%) & 10(76.9\%) & 0 & $4(13.3 \%)$ & 0.012 & $<0.001$ & $<0.001$ \\
\hline$q n r$ & $4(4.8 \%)$ & 0 & $2(5.0 \%)$ & $2(6.70 \%)$ & & & \\
\hline \multicolumn{8}{|l|}{ Adhesins } \\
\hline рарАH & $22(26.5 \%)$ & $12(92.3 \%)$ & $6(15.0 \%)$ & $4(13.3 \%)$ & & $<0.001$ & $<0.001$ \\
\hline papC & $23(27.7 \%)$ & 13(100\%) & $6(15.0 \%)$ & $4(13.3 \%)$ & & $<0.001$ & $<0.001$ \\
\hline papEF & $22(26.5 \%)$ & 12(92.3\%) & $7(17.5 \%)$ & $3(10.0 \%)$ & & $<0.001$ & $<0.001$ \\
\hline papG allele I & 0 & 0 & 0 & 0 & & & \\
\hline papG allele II & $21(25.3 \%)$ & 12(92.3\%) & $6(15.0 \%)$ & $3(10.0 \%)$ & & $<0.001$ & $<0.001$ \\
\hline$s f a / f o c D E$ & $1(1.2 \%)$ & 0 & $1(2.50 \%)$ & 0 & & & \\
\hline focG & 0 & 0 & 0 & 0 & & & \\
\hline$a f a / d r a B C$ & 7 (8.4\%) & 0 & $2(5.0 \%)$ & $5(16.7 \%)$ & & & \\
\hline $\mathrm{fimH}$ & 82 (98.8\%) & 13(100\%) & 39(97.5\%) & $30(100 \%)$ & & & \\
\hline gafD & 0 & 0 & 0 & 0 & & & \\
\hline sfas & 7 (8.4\%) & $3(23.1 \%)$ & $3(7.5 \%)$ & 1(3.3\%) & & & \\
\hline \multicolumn{8}{|l|}{ Toxins } \\
\hline hlyA & 14 (16.9\%) & $11(84.6 \%)$ & $1(2.50 \%)$ & $2(6.70 \%)$ & & $<0.001$ & $<0.001$ \\
\hline$c n f 1$ & 10 (12.0\%) & $8(61.5 \%)$ & $1(2.50 \%)$ & 1(3.3\%) & & 0.002 & $<0.001$ \\
\hline$c d t B$ & 0 & 0 & 0 & 0 & & & \\
\hline $\mathrm{craC}$ & $3(3.6 \%)$ & 0 & 0 & $3(10.0 \%)$ & 0.042 & & \\
\hline
\end{tabular}


Table 1 Characteristics of E. coli ST131 isolates by fimH subtyping (Continued)

\begin{tabular}{|c|c|c|c|c|c|c|c|}
\hline \multirow[t]{3}{*}{ Characteristics } & \multirow{3}{*}{$\begin{array}{l}\text { Total } \\
N=83(\%)\end{array}$} & \multicolumn{3}{|c|}{ ST131 subclones, number of isolates (\%) } & \multicolumn{3}{|l|}{$P^{a}$} \\
\hline & & \multirow{2}{*}{$\begin{array}{l}\text { H3ORx } \\
n=13\end{array}$} & \multirow{2}{*}{$\begin{array}{l}\text { H30 non-Rx } \\
n=40\end{array}$} & \multirow{2}{*}{$\begin{array}{l}\mathrm{H} 41 \\
n=30\end{array}$} & \multirow[t]{2}{*}{$\begin{array}{l}\text { H41 vs } \\
\text { H30 non -Rx }\end{array}$} & \multirow[t]{2}{*}{$\begin{array}{l}\text { H41 vs } \\
\text { H30-Rx }\end{array}$} & \multirow[t]{2}{*}{$\begin{array}{l}\text { H30 Rx vs } \\
\text { H30 non-Rx }\end{array}$} \\
\hline & & & & & & & \\
\hline \multicolumn{8}{|l|}{ Siderophores } \\
\hline fyuA & $81(97.6 \%)$ & 13(100\%) & $38(95.0 \%)$ & $30(100 \%)$ & & & \\
\hline iutA & 77 (92.8\%) & 13(100\%) & $38(95.0 \%)$ & $26(86.7 \%)$ & & & \\
\hline \multicolumn{8}{|l|}{ Capsules } \\
\hline kpsMT /I & $62(74.7 \%)$ & $9(69.2 \%)$ & $33(82.5 \%)$ & $20(66.7 \%)$ & & & \\
\hline kpsMT III & 0 & 0 & 0 & 0 & & & \\
\hline kpsMT K1 & $10(12.0 \%)$ & $2(15.4 \%)$ & $5(12.5 \%)$ & $3(10.0 \%)$ & & & \\
\hline kpsMT K5 & $47(56.6 \%)$ & $11(84.6 \%)$ & $29(72.5 \%)$ & $7(23.3 \%)$ & $<0.001$ & 0.001 & \\
\hline \multicolumn{8}{|l|}{ Miscellaneous } \\
\hline$n f a E$ & 0 & 0 & 0 & 0 & & & \\
\hline$r f c$ & 0 & 0 & 0 & 0 & & & \\
\hline malX & $76(91.6 \%)$ & 13(100\%) & $37(92.5 \%)$ & $26(86.7 \%)$ & & & \\
\hline trat & 73 (88.0\%) & 13(100\%) & $32(80.0 \%)$ & 28(93.3\%) & & & \\
\hline Virulence scores ${ }^{\mathrm{e}}$ & 7 & 9 & 7 & 6 & & 0.001 & 0.001 \\
\hline
\end{tabular}

${ }^{a} P$ values, by either $X^{2}$ or Fisher's exact test or Mann-Whitney test, are shown where $P<0.05$

${ }^{b} E S B L, C T X-M$-type extended-spectrum- $\beta$-lactamase genes

${ }^{\mathrm{C}} \mathrm{FQ}^{\mathrm{R}}$, fluoroquinolone resistance

dGyrA S83 L, D87N, ParC S80I, E84V, and ParE 1529L

emedian number of virulence factors (range)

isolates, and the median age was 62 years (range, 5 years to 89 years).

The results of $\mathrm{O}$ typing revealed that ST131 isolates belonged to two types (O25b and O16). All the 83 ST131 isolates were $\mathrm{O}$-antigen typeable. $\mathrm{O} 25 \mathrm{~b}(66.3 \%)$ was the most prevalent type, followed by $\mathrm{O} 16$ (33.7\%) (Table 1 ). The majority $(52 / 55,94.5 \%)$ of O25b ST131 isolates belonged to fimH30, while $96.4 \%$ (27/28) of O16 ST131 isolates were fimH41subclone. The distribution of $\mathrm{O}$ types was not significantly associated with specimen type or source (Table 2).

Subclone typing showed that $H 30$ lineage comprised $63.9 \%(53 / 83)$ of the ST131 isolates, including 13 (15.7\%) $H 30-\mathrm{Rx}$ and 40 (48.2\%) H30 non-Rx (Table 1). The remaining 30 isolates belonged to fimH41 subclone. Among ciprofloxacin-resistant ST131 isolates, 84.5\% (49/58) belonged to the $H 30$ lineage.

\section{Antimicrobial susceptibility}

Among the 83 ST131 isolates, the highest rates of resistance were to CTX (71.7\%), CIP (69.9\%), LEV (69.9\%) and SXT (67.5\%). On the contrary, resistance rates were low to ATM (36.1\%), FEP (31.3\%), CAZ (26.5\%), AMK (8.4\%) and TZP (1.2\%). None of the isolates was resistant to imipenem or ertapenem. These findings are summarized in Table 1.

Compared with O25b-ST131 isolates, O16-ST131 isolates were shown to have a lower resistance to fluoroquinolones
(CIP and LEV) and FEP, and had a similar resistance to third generation cephalosporins (CTX and CAZ) (Table 2).

CTX-M ESBL and fluoroquinolone resistance mechanisms bla $_{\text {CTX-M }}$ genes were harbored by $62(74.7 \%)$ E. coli ST131 isolates. Of these, 37 isolates carried $b l a_{\text {CTX-M-14, }}$ 22 isolates carried $b l a_{\mathrm{CTX}-\mathrm{M}-15}$ and 1 isolates carried $b l a_{\mathrm{CTX}-\mathrm{M}-123}$. The remaining 2 isolates co-produced bla $_{\mathrm{CTX}-\mathrm{M}-14}$ and $b l a_{\mathrm{CTX}-\mathrm{M}-15}$.

All CTX-M-14 producers were $H 30$ non-Rx and $H 41$, whereas most ST131 H30-Rx subclones were CTX-M15 producers $(92.3 \%, 12 / 13$; Table 1$)$.

All 58 ciprofloxacin-resistant ST131 isolates contained 4 or 5 nonsynonymous mutations in $\operatorname{gyr} A$, parC and parE. All these ST131 isolates had a set of 3 conserved QRDR amino acid substitutions (GyrA S83 L, D87N and ParC S80I). The presence of 5 mutations (GyrA S83 L, D87N, ParC S80I, E84V and ParE I529L) was significantly more prevalent among $H 30$ isolates than $H 41(P<0.001)$. These findings are summarized in Table 1 . Meanwhile, these 5 mutations were strongly associated with $\mathrm{O} 25 \mathrm{~b}$ subclones (Table 3).

Three types of PMQR determinants were found in 16 ST131 isolates, including aac (6')-Ib-cr $(n=14)$, qnrS1 $(n=2)$ and $q n r B 1(\mathrm{n}=2)$. Two ST131 isolates coproduced $q n r B 1$ and $a a c\left(6^{\prime}\right)-I b-c r$. $a a c\left(6^{\prime}\right)-I b-c r$ were significantly concentrated in H30-Rx isolates than non H30-Rx isolates $(P<0.001)$ (Table 1). 
Table 2 The sample sources and distribution of virulence factors among different O-serotype of E. coli ST131 isolates

\begin{tabular}{|c|c|c|c|c|}
\hline \multirow[t]{2}{*}{ Characteristics } & \multicolumn{3}{|c|}{ Clonal groups, number of isolates (\%) } & \multirow{2}{*}{$\begin{array}{l}P^{a} \\
\text { O25b vs } 016\end{array}$} \\
\hline & All isolates $(n=83)$ & O25b $(n=55)$ & $016(n=28)$ & \\
\hline \multicolumn{5}{|l|}{ Source } \\
\hline Urine & $55(66.3 \%)$ & $36(65.5 \%)$ & $19(67.9 \%)$ & \\
\hline Blood & $12(14.5 \%)$ & $10(18.2 \%)$ & $2(7.1 \%)$ & \\
\hline Other & $16(19.3 \%)$ & $9(16.4 \%)$ & $7(25.0 \%)$ & \\
\hline \multicolumn{5}{|c|}{ Antimicrobial resistance } \\
\hline ATM & $30(36.1 \%)$ & $24(43.6 \%)$ & $6(21.4 \%)$ & \\
\hline CAZ & $22(26.5 \%)$ & $16(29.1 \%)$ & $6(21.4 \%)$ & \\
\hline CTX & $59(71.1 \%)$ & $41(74.5 \%)$ & $18(64.3 \%)$ & \\
\hline FEP & $26(31.3 \%)$ & $22(40.0 \%)$ & $4(14.3 \%)$ & 0.024 \\
\hline TZP & $1(1.2 \%)$ & 0 & $1(3.6 \%)$ & \\
\hline $\mathrm{CIP}$ & $58(69.9 \%)$ & $49(89.1 \%)$ & $9(32.1 \%)$ & $<0.001$ \\
\hline LEV & $58(69.9 \%)$ & $49(89.1 \%)$ & $9(32.1 \%)$ & $<0.001$ \\
\hline AMK & $7(8.4 \%)$ & $4(7.3 \%)$ & $3(10.7 \%)$ & \\
\hline SXT & $56(67.5 \%)$ & $36(65.5 \%)$ & $20(71.4 \%)$ & \\
\hline \multicolumn{5}{|l|}{ Adhesins } \\
\hline papAH & $22(26.5 \%)$ & $19(34.5 \%)$ & $3(10.7 \%)$ & 0.034 \\
\hline papC & $23(27.7 \%)$ & $21(38.2 \%)$ & $2(7.1 \%)$ & 0.004 \\
\hline papEF & $22(26.5 \%)$ & 19 (34.5\%) & $3(10.7 \%)$ & 0.034 \\
\hline papG allele I & 0 & 0 & 0 & \\
\hline papG allele II & $21(25.3 \%)$ & $18(32.7 \%)$ & $3(10.7 \%)$ & 0.034 \\
\hline$s f a / f o c D E$ & $1(1.2 \%)$ & $1(1.8 \%)$ & 0 & \\
\hline focG & 0 & 0 & 0 & \\
\hline$a f a / d r a B C$ & 7 (8.4\%) & $2(3.6 \%)$ & $5(17.9 \%)$ & 0.040 \\
\hline $\mathrm{fimH}$ & 82 (98.8\%) & 54 (98.2\%) & 28 (100\%) & \\
\hline gafD & 0 & 0 & 0 & \\
\hline sfas & $7(8.4 \%)$ & $6(10.9 \%)$ & $1(3.6 \%)$ & \\
\hline \multicolumn{5}{|l|}{ Toxins } \\
\hline hlyA & 14 (16.9\%) & 11 (20.0\%) & $3(10.7 \%)$ & \\
\hline cnfl & $10(12.0 \%)$ & $8(14.5 \%)$ & $2(7.1 \%)$ & \\
\hline$c d t B$ & 0 & 0 & 0 & \\
\hline $\mathrm{cvaC}$ & $3(3.6 \%)$ & $1(1.8 \%)$ & $2(7.1 \%)$ & \\
\hline \multicolumn{5}{|l|}{ Siderophores } \\
\hline fyuA & 81 (97.6\%) & $53(96.4 \%)$ & $28(100 \%)$ & \\
\hline iutA & 77 (92.8\%) & $52(94.5 \%)$ & 25 (89.3\%) & \\
\hline \multicolumn{5}{|l|}{ Capsules } \\
\hline kpsMTIl & $62(74.7 \%)$ & $43(78.2 \%)$ & 19 (67.9\%) & \\
\hline kpsMTIII & 0 & 0 & 0 & \\
\hline kpsMT K1 & $10(12.0 \%)$ & $8(14.5 \%)$ & $2(7.1 \%)$ & \\
\hline kpsMT K5 & 47 (56.6\%) & $40(72.7 \%)$ & 7 (25.0\%) & $<0.001$ \\
\hline \multicolumn{5}{|l|}{ Miscellaneous } \\
\hline$n f a E$ & 0 & 0 & 0 & \\
\hline$r f c$ & 0 & 0 & 0 & \\
\hline
\end{tabular}


Table 2 The sample sources and distribution of virulence factors among different O-serotype of E. coli ST131 isolates (Continued)

\begin{tabular}{|c|c|c|c|c|}
\hline \multirow[t]{2}{*}{ Characteristics } & \multicolumn{3}{|c|}{ Clonal groups, number of isolates (\%) } & \multirow{2}{*}{$\begin{array}{l}P^{a} \\
\text { O25b vs } 016\end{array}$} \\
\hline & All isolates $(n=83)$ & $\mathrm{O} 25 \mathrm{~b}(n=55)$ & $016(n=28)$ & \\
\hline malx & $76(91.6 \%)$ & $51(92.7 \%)$ & $25(89.3 \%)$ & \\
\hline trat & $73(88.0 \%)$ & $46(83.6 \%)$ & $27(96.4 \%)$ & \\
\hline Virulence scores ${ }^{\mathrm{b}}$ & 7 (3 to 11$)$ & $7(3$ to 11$)$ & $6(4$ to 10$)$ & \\
\hline
\end{tabular}

${ }^{a} P$ values, by either $X^{2}$ or Fisher's exact test, are shown where $P<0.05$

${ }^{b}$ median number of virulence factors (range)

\section{Virulence factors}

The most frequent VF genes were fimH (type 1 fimbriae), fyuA (yersiniabactin), iutA (iron uptake gene), malX (pathogenicity-associated island marker), kpsMT II (group II capsule), and traT (outer membrane lipoprotein), each of which was detected in $\geq 60 \%$ of the isolates. In contrast, 8 genes were each identified in less than $10 \%$ of isolates, including $\mathrm{cvaC}$, sfa/focDE, sfaS, afa/draBC, focG, papG allele I, gafD, $c d t B$, kpsMTIII, $n f a E$ and $r f c$. The median virulence score was 7 (range, 3 to 11). Among the two $O$ groups, the median virulence scores (ranges) were 7 (3 to 11) for O25b-ST131 and 6 (4 to 10) for O16-ST131, respectively.

ST131 H30-Rx isolates possessed the highest virulence score (mean, 9; range, 7 to11). ST131 H30 non-Rx and $H 41$ isolates showed similar virulence scores and ranges of VFs (mean scores, 7 [range, 4 to 9] and 6 [range, 3 to 9], respectively). ST131 H30-Rx isolates had significantly higher virulence score than $H 30$ non-Rx and $H 41$ isolates (Table 1). Four adhesins (papAH, papC, papEF and papG allele II) and two toxins ( $h l y A$ and $\operatorname{cnf} 1$ ) were significantly more frequent among ST131 H30-Rx isolates (Table 1).

\section{Bacterial clonal relatedness}

PFGE analysis of the 83 E. coli ST131 isolates demonstrated that these isolates were classified into 68 pulsotypes (named 1-68, Fig. 1) and these subtypes were grouped into 23 different PFGE clusters (named A-W, in Fig. 1) using 70\% similarity cut-off value. Two PFGE clusters (C and D) were predominant, grouping 13 and 14 isolates, respectively, whereas the other 21 PFGE clusters contained up to 9 ST131 isolates each (Fig. 1). Cluster C contained 92.3\% (12/ 13) of H30-Rx isolates. The majority of O16 ST131 isolates belonged to two clusters ( $\mathrm{H}$ and $\mathrm{I}$ ), grouping 5 and 9 isolates. As shown in Fig. 1, at 63\% similarity level, O25bST131 isolates were clustered into six major clonal groups, while $92.9 \%$ of the $\mathrm{O} 16$ isolates were clustered into two clonal groups (group one contained clusters $\mathrm{H}, \mathrm{I}$, J and $\mathrm{K}$, and group two contained clusters $\mathrm{O}, \mathrm{P}, \mathrm{Q}, \mathrm{R}$ and $\mathrm{S}$ ).

\section{Discussion}

To our knowledge, this is the first molecular survey reporting the prevalence and characteristics of E. coli ST131 and its subclones among unselected clinical isolates in China. We found E. coli ST131 accounted for $11.6 \%$ of all $E$. coli isolates, which is similar to rates reported previously [1, 22, 23]. In addition, there was a similar distribution of ST131 among urine and blood samples. Meanwhile, the PFGE study indicated that ST131 isolates in this study were highly diverse at the pulsotype level and there was no predominated clone (Fig. 1).

O25b was previously considered the predominant ST131 clone type, while $\mathrm{O} 16$ isolates accounted for a small percentage $[1,2,24]$. Similar to previous studies [23, 24],

Table 3 Fluoroquinolone resistance mechanisms, O types and fimH subclone among 83 ST131 E. coli isolates

\begin{tabular}{|c|c|c|c|c|c|c|c|c|}
\hline$\overline{C I P^{a}}$ & fimH type (n) & O type $(n)$ & $\mathrm{N}$ & gyrA & parC & parE & aac(6')-lb-cr & qnr (n) \\
\hline $\mathrm{R}$ & $\mathrm{H} 30$ & $\mathrm{O} 25 \mathrm{~b}$ & 48 & S83 L, D87N & S80I, E84V & I529L & 9 & anrS1(1) \\
\hline$R$ & $\mathrm{H} 41$ & 016 & 3 & S83 L, D87N & S80I, E84V & I529L & 1 & $\operatorname{anrB1}(1)$ \\
\hline $\mathrm{R}$ & $\mathrm{H} 30$ & $\mathrm{O} 25 \mathrm{~b}$ & 1 & S83 L, D87N & S801 & P415L & - & - \\
\hline R & $\mathrm{H} 41$ & 016 & 1 & S83 L, D87N & S80I & I529L & - & - \\
\hline R & $\mathrm{H} 41$ & 016 & 1 & S83 L, D87N & S80I & S458A & 1 & - \\
\hline R & $\mathrm{H} 41$ & 016 & 3 & S83 L, D87N & S80I & $\llcorner 445 \mathrm{H}$ & 1 & - \\
\hline R & $\mathrm{H} 41$ & $\mathrm{O} 25 \mathrm{~b}$ & 1 & S83 L, D87N & S80I & $\llcorner 445 \mathrm{H}$ & - & - \\
\hline । & $\mathrm{H} 41$ & O25b & 1 & $N D^{b}$ & ND & ND & 1 & $\operatorname{anrB1(1)}$ \\
\hline S & $\mathrm{H} 41$ & 016 & 19 & ND & ND & ND & 1 & - \\
\hline S & $\mathrm{H} 41$ & $\mathrm{O} 25 \mathrm{~b}$ & 2 & ND & ND & ND & - & $\operatorname{anrS1}(1)^{c}$ \\
\hline S & $\mathrm{H} 30$ & $\mathrm{O} 25 \mathrm{~b}$ & 3 & ND & ND & ND & - & - \\
\hline
\end{tabular}

${ }^{a} R$, resistant; I, intermediate; $S$, susceptible

${ }^{\mathrm{b}} \mathrm{ND}$, not detected

${ }^{\mathrm{c}}$ In this isolate, $b / a_{\mathrm{CTX}-\mathrm{M}-15}$ was positive 


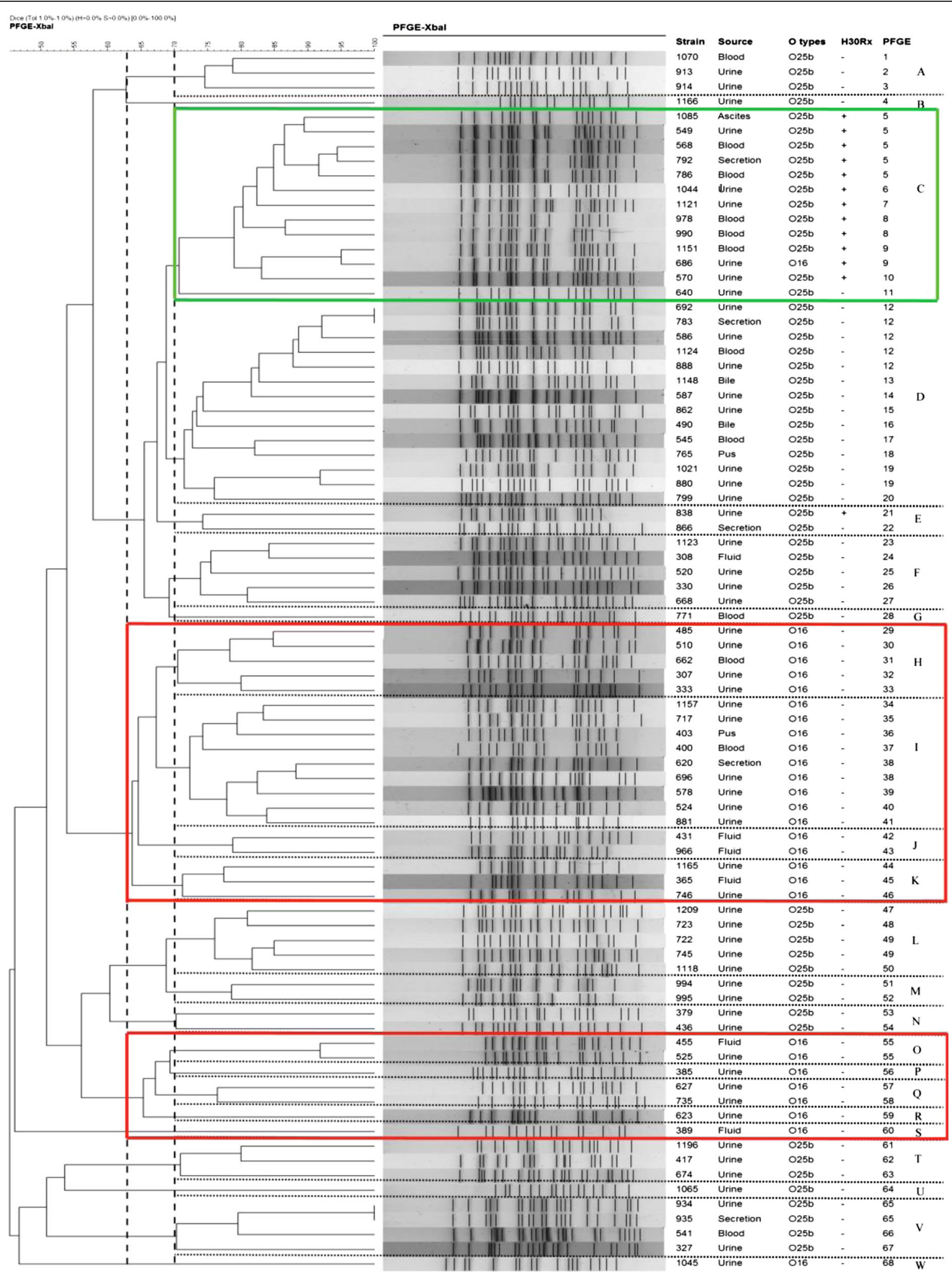

Fig. 1 Pulsed-field gel electrophoresis (PFGE) of Xbal-digested DNA from 83 ST131 E. coli isolates. Strain designation, Sample source, O serotype, H30 Rx and PFGE patterns are shown. Green box indicated the main cluster of ST131 H30 Rx isolates using 70\% similarity cut-off value. Red box showed the two main clusters of O16 ST131 isolates at 63\% similarity level 
O25b-B2-ST131 in this study was the predominant clone among clinical E. coli isolates. Notably, a high percentage (33.6\%) of O16-B2-ST131 was found in the present study. In China, a recent study found that O16-B2ST131 accounted for the predominant subset among ST131 fecal E. coli isolates [25]. However, among E. coli clinical isolates, all other researches focused on O25bB2-ST131 in our country and no data are available on the prevalence of O16-B2-ST131 among E. coli clinical isolates till now, the present study is the first to show the presence of O16-B2-ST131 in this country. Taken together with the previous study [25], these findings suggest that O16-B2-ST131 may emerge as an important type of E. coli ST131 in China. The rate of O25b-B2-ST131 was not striking in this study. However, the O16-B2-ST131 may need special attention for the high percentages found in our results and previous study [25]. As discussed before, the clonal group should be investigated in the future study in other geographical regions in China $[25,26]$.

The $H 30$ lineage was a very important ST131 subclone, which firstly appeared in the year of 2000 and expanded rapidly to become the most dominant and extensively distributed multidrug-resistant lineage of $E$. coli worldwide $[1,2]$. Within the $H 30$ subclone, the $H 30-\mathrm{Rx}$ subset is a major drug-resistant pathogen among fluoroquinoloneresistant $E$. coli isolates and is associated with $b l a_{\mathrm{CTX}-\mathrm{M}-15}$ $[1,2]$. In this study, we also explored the prevalence of $H 30$ lineage and H30-Rx sublineage among E. coli ST131 isolates. H30 lineage accounted for the majority of ST131 isolates in our study, which is similar to those in previous studies [22, 23]. However, the prevalence of H30-Rx ST131 was obviously lower than those reported in other researches $[23,27]$. Meanwhile, the great majority of $H 30-\mathrm{Rx}$ isolates harbored $b l a_{\mathrm{CTX}-\mathrm{M}-15}$ in this study. Resistances to 11 most commonly used antimicrobials were evaluated in this study. Our findings support the strong association of H30-Rx sublineage with multidrug resistance and the presence of $b l a_{\mathrm{CTX}-\mathrm{M}-15}$ (Table 1). We also identified the H30-Rx with the presence of $a a c\left(6^{\prime}\right)-I b-c r$ (Table 1). Resistance of fluoroquinolones is mainly due to chromosomal mutations in the QRDR regions, especially in gyrA and parC $[28,29]$. In this study, A set of 3 conserved mutations in QRDR regions (GyrA S83 L, D87N, and ParC S80I) could be found in all the CIP-resistant ST131 isolates. These chromosomal mutations have been previously linked to fluoroquinolone resistance $[23,30]$. Meanwhile, 5 mutations (GyrA S83 L, D87N, ParC S80I, E84V and ParE I529L) were strongly associated with $H 30$ ST131 isolates, which is similar to the previous studies [23, 31].

Regarding virulence factors, fimH, fyuA, iutA, kpsMTII and traT were the most prevalent virulence factors found in this study, which have been associated with E. coli ST131 isolates in previous studies $[1,32]$. There was a similar prevalence rate for these five virulence factors within different O types ST131 isolates (Table 2). O16-B2-ST131 isolates harbored similar number of virulence genes than O25b-ST131 isolates. Compared with O25b-B2-ST131 isolates, O16-B2-ST131 isolates harbored less virulence factors of adhesins (papAH, papC, papEF and papG alleleII).The pap adhesion genes probably played an important role in the pathophysiology of pyelonephritis caused by E. coli $[33,34]$. This property probably suggests that O16-B2ST131 isolates have a lower adhesive ability and pathogenicity than O25b-B2-ST131. Meanwhile, O25b-B2-ST131 isolates were significantly more likely to possess kpsMT K5 than O16-B2-ST131 isolates. kpsMT K5 is a capsule synthesis gene [16]. The possession of the gene may enhance the pathogenesis of O25b-B2-ST131 isolates due to the evasion of phagocytosis (capsule encoded by kpsMT K5).

In conclusion, to our knowledge, this is the first report on the prevalence and molecular characteristic of ST131 clonal group among consecutive clinical $E$. coli isolates in China. Our findings demonstrated that ST131 lineage accounts for a small proportion of clinical $E$. coli isolates in China, which included two major groups: O25b-B2ST131 and O16-B2-ST131. Our results implies that O16B2-ST131 subclone may be an important type of E. coli ST 131 in China, which suggests that future studies should not ignore such clone in this country.

\section{Abbreviations \\ E. coll: Escherichia coli; ESBL: Extended-spectrum $\beta$-lactamases; MLST: multilocus sequence typing; QRDR: Quinolone-resistance determining region; PFGE: \\ Pulsed-field gel electrophoresis analysis; ST: Sequence type; VF: Virulence score \\ Acknowledgements \\ The authors thank Prof. Yohei Doi, University of Pittsburgh School of Medicine (USA) for his useful discussion and for his critical review of the manuscript.We also thank Prof. Hu Fupin, Institute of Antibiotics, Huashan Hospital, for his kindly help in performing PFGE experiment.}

\section{Funding}

This study was supported by the Fujian Provincial Funds for Distinguished Young Scientists in Colleges and Universities, China (grant no. JA13134), and the Medical Elite Cultivation Program of Fujian, China (grant no. 2015-ZQN-ZD-15).

Availability of data and materials

All the data supporting the findings are presented in the manuscript.

Authors' contributions

$\mathrm{BL}$ and $\mathrm{YC}$ designed the experiment, $\mathrm{BL}$ wrote the manuscript. $\mathrm{YL}, \mathrm{FL}$ and $\mathrm{QH}$ performed the experiments. $\mathrm{CL}$ participated in data analysis. YC reviewed the draft. All authors read and approved the final manuscript.

Ethics approval and consent to participate

Not applicable.

Consent for publication

Not applicable.

Competing interests

The authors declare that they have no competing interests.

\section{Publisher's Note}

Springer Nature remains neutral with regard to jurisdictional claims in published maps and institutional affiliations. 


\section{Author details}

'Department of Clinical Laboratory, Fujian Medical University Union Hospital, 29 Xinquan Rd, Fuzhou, Fujian 350001, People's Republic of China. ${ }^{2}$ Department of Clinical Laboratory, Maternal and Children's Health Hospital of Fujian Province, Fuzhou, Fujian 350001, China. ${ }^{3}$ Department of Infectious Disease, Fujian Medical University Union Hospital, Fuzhou, Fujian 350001, China.

Received: 29 September 2017 Accepted: 8 November 2017 Published online: 15 November 2017

\section{References}

1. Nicolas-Chanoine MH, Bertrand X, Madec JY. Escherichia Coli ST131, an intriguing clonal group. Clin Microbiol Rev. 2014;27:543-74.

2. Mathers AJ, Peirano G, Pitout JD. The role of epidemic resistance plasmids and international high-risk clones in the spread of multidrug-resistant Enterobacteriaceae. Clin MicrobioRev. 2015;28:565-91.

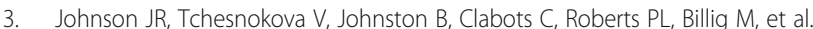
Abrupt emergence of single dominant multidrug-resistant strain of Escherichia Coli. J Infect Dis. 2013;207:919-28.

4. Sauget M, Cholley P, Vannier A, Thouverez M, Nicolas-Chanoine MH, Hocquet $D$, et al. Trends of extended-spectrum $\beta$-lactamase-producing Escherichia Coli sequence type 131 and its H30 subclone in a French hospital over a 15-year period. Int J Antimicrob Agents. 2016;48:744-7.

5. Matsumura Y, Johnson JR, Yamamoto M, Nagao M, Tanaka M, Takakura S, et al. CTX-M-27- and CTX-M-14-producing, ciprofloxacin-resistant Escherichia Coli of the H30 subclonal group within ST131 drive a Japanese regional ESBL epidemic. J Antimicrob Chemother. 2015;70:1639-49.

6. Ben Zakour NL, Alsheikh-Hussain AS, Ashcroft MM, Khanh Nhu NT, Roberts LW, Stanton-Cook M, et al. Sequential Acquisition of Virulence and Fluoroquinolone Resistance has Shaped the evolution of Escherichia Coli ST131. MBio. 2016;7:e00347-16.

7. Wang LH, Liu PP, Wei DD, Liu Y, Wan LG, Xiang TX, et al. Clinical isolates of uropathogenic Escherichia Coli ST131 producing NDM-7 metallo- $\beta$ lactamase inChina. Int J Antimicrob Agents. 2016;48:41-5.

8. Zhang F, Zhu D, Xie L, Guo X, Ni Y, Sun J. Molecular epidemiology of carbapenemase-producing Escherichia Coli and the prevalence of ST131 subclone H30 in shanghai, China. Eur J Clin Microbiol Infect Dis. 2015;34:1263-9.

9. Wang S, Zhao SY, Xiao SZ, FF G, Liu QZ, Tang J, et al. Antimicrobial resistance and molecular epidemiology of Escherichia Coli causing bloodstream infections in three hospitals in shanghai, China. PLoS One. 2016;11:e0147740

10. Johnson JR, Menard M, Johnston B, Kuskowski MA, Nichol K, Zhanel GG. Epidemic clonal groups of Escherichia Coli as a cause of antimicrobialresistant urinary tract infections in Canada, 2002 to 2004. Antimicrob Agents Chemother. 2009;53:2733-9.

11. Colpan A, Johnston B, Porter S, Clabots C, Anway R, Thao L, et al. Escherichia coli sequence type 131 (ST131) subclone H30 as an emergent multidrugresistant pathogen among US veterans. Clin Infect Dis. 2013;57:1256-65.

12. Clinical and Laboratory Standards Institute. Performance Standards for Antimicrobial Susceptibility Testing; 24th Informational Supplement, M100S26. PA: Wayne; 2016.

13. Clermont O, Christenson JK, Denamur E, Gordon DM. The Clermont Escherichia coli phylo-typing method revisited: improvement of specificity anddetection of new phylo-groups. Environ Microbiol Rep. 2013;5:58-65.

14. Li D, Liu B, Chen M, Guo D, Guo X, Liu F, et al. A multiplex PCR method to detect 14 Escherichia coli serogroups associated with urinary tract infections. Jicrobiol Methods. 2010;82:71-7.

15. Clermont O, Dhanji H, Upton M, Gibreel T, Fox A, Boyd D, et al. Rapid detection of the O25b-ST131 clone of Escherichia coli encompassing the CTX-M-15-producing strains. J Antimicrob Chemother. 2009;64:274-7.

16. Johnson JR, Stell AL. Extended virulence genotypes of Escherichia coli strains from patients with urosepsis in relation to phylogeny and host compromise. J Infect Dis. 2000;181:261-72.

17. Oteo J, Gonzalez-Lopez JJ, Ortega A, Quintero-Zarate JN, Bou G, Cercenada E, et al. Inhibitor-resistant TEM- and OXA-1- producing Escherichia Coli isolates resistant to amoxicillin-clavulanate are more clonal and possess lower virulence gene content than susceptible clinical isolates. Antimicrob Agents Chemother. 2014;58:3874-81.
18. Li B, Sun JY, Liu QZ, Han LZ, Huang XH, Ni YX. High prevalence of CTX-M $\beta$ lactamases in faecal Escherichia coli strains from healthy humans in Fuzhou, China. Scand J Infect Dis. 2011;43:170-4.

19. Komp Lindgren $P$, Karlsson A, Hughes D. Mutation rate and evolution of fluoroquinolone resistance in Escherichia Coli isolates from patients with urinary tract infections. Antimicrob Agents Chemother. 2003;47:3222-32.

20. Robicsek A, Strahilevitz J, Jacoby GA, Macielag M, Abbanat D, Park CH, et al. Fluoroquinolone-modifying enzyme: a new adaptation of a common aminoglycoside acetyltransferase. Nat Med. 2006;12:83-8.

21. Yamane K, Wachino J, Suzuki S, Kimura K, Shibata N, Kato H, et al. New plasmid-mediated fluoroquinolone efflux pump, QepA, found in an Escherichia coli clinical isolate. Antimicrob Agents Chemother. 2007;51:3354-60.

22. Lafolie J, Nicolas-Chanoine MH, Grenouillet F, Hocquet D, Bertrand X Prevalence of Escherichia coli sequence type 131 and its H30 subclone among E. coli isolates in a French hospital. Int J Antimicrob Agents. 2014:44:466-8.

23. Kim SY, Park YJ, Johnson JR, JK Y, Kim YK, Kim YS. Prevalence and characteristics of Escherichia coli sequence type 131 and its H3O and H3ORX subclones: a multicenter study from Korea. Diagn Microbiol Infect Dis. 2016;84:97-101.

24. Banerjee R, Johnson JRA. New clone sweeps clean: the enigmatic emergence of Escherichia coli sequence type 131. Antimicrob Agents Chemother. 2014;58: 4997-5004.

25. Zhong YM, Liu WE, Liang XH, Li YM, Jian ZJ, Hawkey PM. Emergence and spread of O16-ST131 and O25b-ST131 clones among faecal CTX-Mproducing Escherichia Coli in healthy individuals in Hunan Province, China. J Antimicrob Chemother. 2015:70:2223-7.

26. Matsumura $Y$, Yamamoto $M$, Nagao M, Hotta G, Matsushima A, Ito $Y$, et al. Emergence and spread of B2-ST131-O25b, B2-ST131-O16 and D-ST405 clonal groups among extended-spectrum- $\beta$-lactamase-producing Escherichia coli in Japan. J Antimicrob Chemother. 2012;67:2612-20.

27. Peirano G, van der Bij AK, Freeman JL, Poirel L, Nordmann P, Costello M, et al. Characheristics of Escherichia Coli sequence type 131 isolates that produce extended-spectrum $\beta$-lactamases: global distribution of the $\mathrm{H} 30-\mathrm{Rx}$ sublineage. Antimicrob Agents Chemother. 2014;58:3762-7.

28. Hooper DC, Jacoby GA. Mechanisms of drug resistance: quinolone resistance. Ann N Y Acad Sci. 2015;1354:12-31.

29. Aldred KJ, Kerns RJ, Osheroff N. Mechanism of quinolone action and resistance. Biochemistry. 2014;53:1565-74.

30. Johnning A, Kristiansson E, Angelin M, Marathe N, Shouche YS, Johansson A et al. Quinolone resistance mutations in the faecal microbiota of Swedish travellers to India. BMC Microbiol. 2015;15:235.

31. Johnson JR, Johnston B, Kuskowski MA, Sokurenko EV, Tchesnokova V. Intensity and mechanisms of fluoroquinolone resistance within the $\mathrm{H} 30$ and H30Rx subclones of Escherichia Coli sequence type 131 compared with other fluoroquinolone-resistant E. Coli. Antimicrob Agents Chemother. 2015:59:4471-80.

32. Olesen B, Frimodt-Møller J, Leihof RF, Struve C, Johnston B, Hansen DS, et al. Temporal trends in antimicrobial resistance and virulence-associated traits within the Escherichia Coli sequence type 131 clonal group and its H30 and H30-Rx subclones, 1968 to 2012. Antimicrob Agents Chemother. 2014;58:6886-95.

33. Farrell DJ, Morrissey I, De Rubeis D, Robbins M, Felmingham DAUK. Multicentre study and the antimicrobial susceptibility of bacterial pathogens causing urinary tract infection. J Inf Secur. 2003;46:94-100.

34. Tarchouna M, Ferjani A, Ben-Selma W, Boukadida J. Distribution of uropathogenic virulence genes in Escherichia coli isolated from patients with urinary tract infection. Int J InfectDis. 2013;17:e450-3. 